\title{
Los indicadores de gestión hospitalaria en tiempos de Covid 19
}

\author{
Hospital management indicators in times of Covid 19 \\ Yasniel Sánchez Suárez. ${ }^{1}$, Leydis Trujillo García. ${ }^{2}$, Maylín Marqués León. ${ }^{3}$ \& \\ Orlando Santos Pérez. ${ }^{4}$
}

DOI: https://doi.org/10.33262/visionariodigital.v5i4.1901

\begin{abstract}
Introduction: Hospital organizations play an outstanding role in the treatment and cure of people with the most serious conditions. With the onset of the pandemic caused by covid 19, the hospital must worry about carrying out research in the biosocial, epidemiological and health fields in order to increase the competitiveness of its management. Objective: to carry out a bibliographic analysis on the main aspects of hospital management in times of covid 19. Results: A summary of the most relevant aspects of hospital management in times of covid 19 is obtained, the main methods of grouping patients used, The most widely used patient classification method is structured triage and Diagnosis Related Groups, the main elements of the new integrated hospital management are determined, in order to optimize the service and the main hospital management indicators as a tool that allows generating timely and reliable information to guide the institution towards the achievement of its established objectives. Conclusions: The new integrated management system contains three main pillars: epidemiological management, preventive services and case surveillance, for which alternatives such as active research and epidemiological surveys, supported by computer applications, have been used.
\end{abstract}

\footnotetext{
${ }^{1}$ Adiestrado de la Carrera Ingeniería Industrial de la Universidad de Matanzas, Cuba. Correo electrónico yasnielsanchez9707@gmail.com

${ }^{2}$ Estudiante de la Carrera Ingeniería Industrial de la Universidad de Matanzas, Cuba. Correo electrónico trujilloleydis99@gmail.com

${ }^{3}$ Doctora en Ciencias Técnicas. Profesora Titular de la Universidad de Matanzas. Teléfono: (+53) (45) 266904 Correo: maylin.marques@umcc.cu

${ }^{4}$ Ingeniero Civil, Máster en Gestión de la Producción y los Servicios, Máster en Docencia Universitaria e Investigación Educativa, y Doctor en Ciencias Técnicas, Profesor Auxiliar. Empresa de Proyectos de Arquitectura e Ingeniería de Matanzas. Email: orlando-santos@empai.cu
} 
Keywords: hospital casuistry; covid 19; hospital management; health services, hospital indicators

\section{Resumen}

Introducción: Las organizaciones hospitalarias cumplen un rol sobresaliente en el tratamiento y curación de las personas con las afecciones más serias. Con la aparición de la pandemia provocada por la covid 19, el hospital debe preocuparse por llevar a cabo investigaciones en el campo biosocial, epidemiológico y sanitario para así aumentar la competitividad de su gestión. Objetivo: realizar un análisis bibliográfico sobre los principales aspectos de la gestión hospitalaria en tiempos de covid 19. Resultados: Se obtiene un resumen de los aspectos más relevantes de la gestión hospitalaria en tiempos de covid 19, los principales métodos de agrupación de pacientes utilizados, el método de clasificación de pacientes más utilizado es el triaje estructurado y los Grupos Relacionados de Diagnóstico, se determinan los principales elementos de la nueva gestión integrada hospitalaria, con el fin de optimizar el servicio y los principales indicadores de gestión hospitalaria como herramienta que permite generar información oportuna y confiable para guiar a la institución hacia la consecución de sus objetivos establecidos. Conclusiones: El nuevo sistema de gestión integrada contiene tres pilares principales la gestión epidemiológica, de servicios preventivos y vigilancia de caso, para lo que se ha empleado alternativas como la pesquisa activa y las encuestas epidemiológicas, apoyadas en aplicaciones informáticas.

Palabras claves: casuística hospitalaria; covid 19; gestión hospitalaria; servicios de salud, indicadores hospitalarios

\section{Introducción}

Los servicios de salud bajo un enfoque integral (Terán Rosero et al. 2019) son un bien público que enfrentan complejos retos y tienen en cuenta la relación entre la salud, el desarrollo social y económico, con una lógica de búsqueda de ventajas comparativas en un sistema internacional abierto y altamente competitivo (OPS 2017, Sánchez Torres 2017).

La organización de salud se debe entender como un conjunto de procesos que producen resultados de valor para los pacientes, más que como un conjunto de funciones separadas entre sí y que tienden a optimizar sus resultados, sin tener en cuenta los objetivos finales de la organización y del sistema de salud (Marqués León 2013).

En este sentido, la atención sobre la promoción de la salud, bien entendida, en su nivel local y comunitario, es más fruto del esfuerzo de técnicos comprometidos que de programas institucionales puestos en valor por las autoridades sanitarias (Hernández Aguado and García 2021). Según (Peñafiel Chang, Camelli, and Peñafiel Chang 2020) la Covid 19 ha impuesto a la región de las américas varios talones de Aquiles, entre ellos el endeudamiento, la crisis migratoria, la creciente desafección, elevados niveles de 
corrupción, la desaceleración y estancamiento económico acumulado, la fuga de capitales, la devaluación de las monedas y sobre todo la carencia en infraestructura sanitaria (OIT 2019), lo que afecta de manera directa los sistemas de calidad de salud.

La salud se reconoce en Cuba como una de sus principales conquistas; el alcance de altos niveles de salud y calidad de vida es uno de los logros de mayor repercusión política y social del país, y su prestigio sobrepasa las fronteras nacionales (Hernández Nariño 2010).

Cuba, a pesar de ser un país subdesarrollado y bloqueado económicamente por los Estados Unidos, posee uno de los mejores sistemas de salud de América Latina, el Sistema Nacional de Salud (SNS) dispone de 161 hospitales, 110 salas de terapia intensiva, 120 áreas intensivas municipales, 449 policlínicos, 111 clínicas estomatológicas, 132 hogares maternos, 12 institutos de investigación, 680 bibliotecas médicas, 155 hogares de ancianos, 295 casas de abuelos, 52 servicios de geriatría y 30 centros médicos psicopedagógico (MSP 2019). En el nuevo contexto de la Covid 19 y como parte de la estrategia nacional diseñada para la contención de la pandemia se disponen de 22 laboratorios de Biología Molecular, tras una inversión que supera el millón de pesos (León Moyal 2021).

Según Hernández Nariño (2010) dentro de los servicios de salud en Cuba, las organizaciones hospitalarias cumplen un rol sobresaliente en el tratamiento y curación de las personas con las afecciones más serias, lo cual demanda una atención especializada y tecnológicamente avanzada. En cifras esta situación se sustenta en el hecho que estas organizaciones consumen el $90 \%$ del gasto de salud.

La gestión de proyectos hospitalarios, como aspecto fundamental, del servicio que se brinda a la comunidad, se debería ejecutar a través de políticas, sistemas, componentes, procesos e instrumentos que al integrarse en forma coherente, holística e integral, como consecuencia, establezcan una prestación de servicios de salud de calidad, permitiendo una gestión dirigida al logro de los objetivos sanitarios y económicos en el marco de una red de servicios de salud eficiente (Ferreira Junior and Porto 2018).

La gestión hospitalaria actual en la atención de pacientes con Covid 19 implica precauciones de aislamiento, limitación de visitas familiares o incluso contacto físico limitado con el personal del hospital. Además del uso de elementos de protección personal por parte del personal del hospital y su entorno, que son una medida importante de bioseguridad con evidencia demostrada, despersonaliza y puede ser aterrador desde la perspectiva del paciente (Chavarro Carvajal et al. 2020).

En una situación de pandemia, los flujos de pacientes hacia el sistema sanitario seguirán un patrón determinado caracterizado por un aumento progresivo de las demandas asistenciales. Este aumento de demanda seguirá el patrón de la curva epidémica que se vaya produciendo (Castro Delgado, Arcos González, and Rodríguez Soler 2009).

La nueva situación internacional provocada por la covid 19 demanda de hospitales y sistemas de salud más organizados que permitan garantizar la calidad del servicio a los 
pacientes y la adaptabilidad temprana a las nuevas condiciones. Las nuevas estrategias adoptadas por las direcciones hospitalarias son de gran importancia a fin de homogenizar y estandarizar nuevas prácticas en el accionar de los sistemas de salud a nivel mundial, regional y local. El objetivo del presente artículo es realizar un análisis bibliográfico sobre los principales aspectos de la gestión hospitalaria en tiempos de covid 19.

\section{Métodos}

Se realiza una revisión documental en revistas indexadas en las bases de datos (BD) Scopus, Scielo, DOAJ y Redalic, Google académico y Sciencedirect.

En la estrategia de búsqueda empleada en la revisión documental se utilizan inicialmente las palabras claves: "gestión hospitalaria", "servicios de salud", "indicadores hospitalarios", "casuística hospitalaria", "gestión hospitalaria en tiempos de covid-19", sin restricciones idiomáticas. Se realiza un análisis exhaustivo de los artículos, se analizaron la relevancia de las citas de los trabajos consultados.

\section{Resultados y Discusión}

Se realiza una revisión de la bibliográfica tanto a nivel nacional e internacional sobre los principales conceptos de gestión hospitalaria en tiempos de covid-19, así como del uso de indicadores de gestión.

\section{La gestión hospitalaria como eje sustancial del sistema de salud}

Clásicamente el hospital se consideró como una institución cerrada y específica del tratamiento del enfermo; pero poco a poco tal criterio se ha ido modificando, en la actualidad el hospital debe ser una institución abierta, dinámica, extramural, en la cual se realizan en forma simultánea y con el mismo interés, funciones tanto preventivas como curativas y su competitividad (Figura 1) depende de varios factores.

El hospital debe preocuparse por llevar a cabo investigaciones en el campo biosocial, epidemiológico y sanitario. La labor educacional debe constituir parte importante de su trabajo diario y estar focalizada en decisiones de política pública y en aseguramiento de las condiciones de salubridad y calidad del servicio hacia los pacientes (Morales Carrillo et al. 2018). La educación proyecta en forma positiva el acto propiamente profesional (Amador Guevara 1979).

Hernández Nariño (2010) en concordancia con (González Pérez 1997) plantea que el servicio hospitalario posee las peculiaridades siguientes:

- $\quad$ El cliente es el paciente y su acompañante

- $\quad$ No existe límite de recursos a utilizarse en cada caso

- Uno de los resultados del proceso es el paciente tratado y curado

- $\quad$ El cliente no decide que va a comprar, no paga el producto o servicio que recibe, pero sí evalúa la calidad percibida, y percibe mayor calidad del servicio cuanto más tiempo se invierta en su atención 


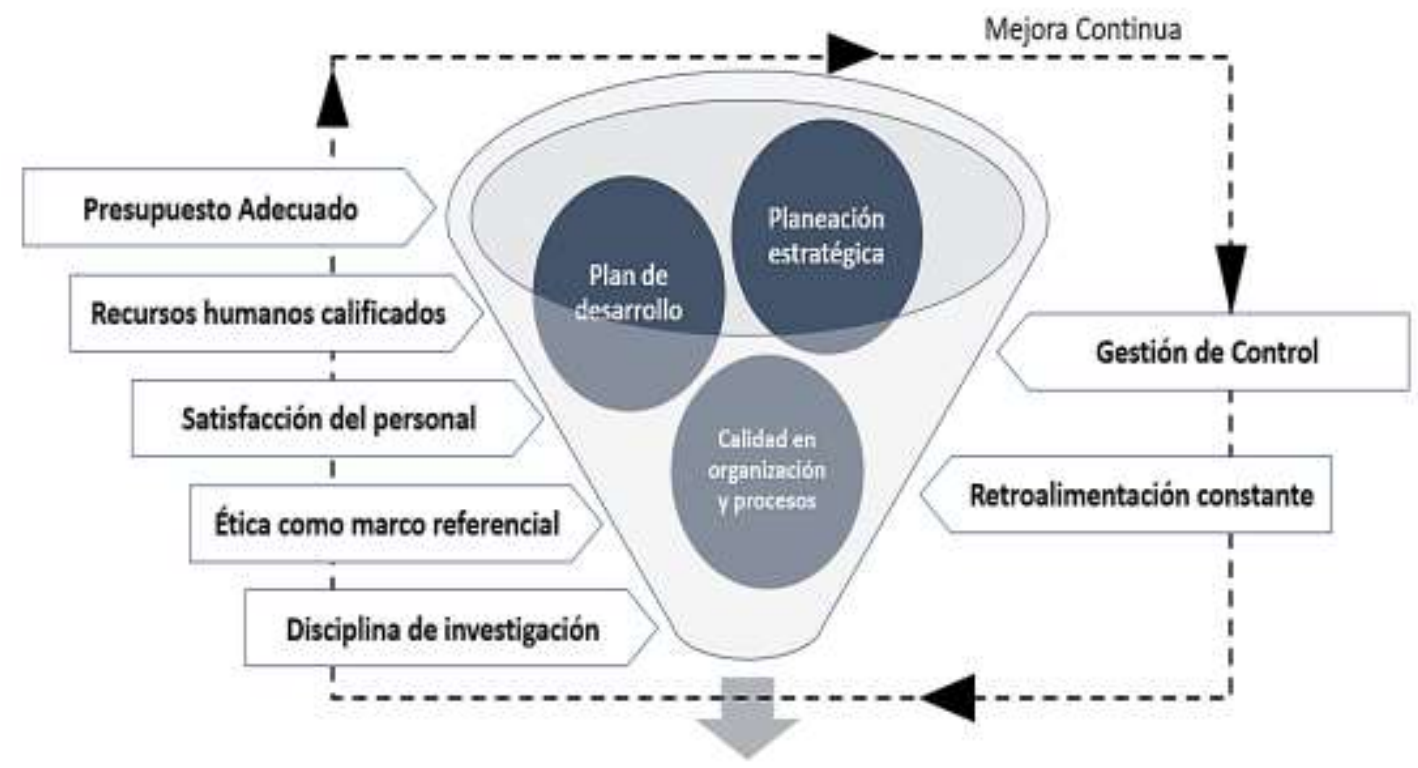

\section{Competitividad Hospitalaria}

Figura 1. Posibilidad competitiva hospitalaria.

Fuente: elaboración propia.

La clasificación de hospitales es de gran importancia para la organización y planificación de los servicios de salud (Fuentelsaz Gallego et al. 2012), además de facilitar su estudio, caracterización y diagnóstico, y aportar soluciones y herramientas de trabajo acordes a las características de cada instalación hospitalaria (Hernández Nariño 2010).

En los hospitales, como en todas las organizaciones, existe la necesidad de optimizar los recursos disponibles (Suárez Barraza 2020) y de lograr su uso racional y productivo, donde se pone de manifiesto lo indispensable que resulta el empleo de métodos efectivos para la planificación de las operaciones. La consideración dominante en el diseño, planeación, control, análisis y administración de estas operaciones de servicios es la aplicación de las habilidades y tecnologías médicas. Como los casos de los pacientes son tan distintos, los hospitales deben ser lo suficientemente flexibles como para aceptar una amplia variedad de tipos y secuencias de tratamiento para los pacientes (Marqués León 2013).

A partir de los conceptos y especificaciones realizadas, el autor resume la gestión hospitalaria como la planificación, organización, análisis y control de los procesos hospitalarios, necesarios para el logro de la competitividad hospitalaria con una visión abierta, dinámica, extramural, donde las funciones preventivas y curativas se encuentren alineadas para garantizar la satisfacción de los pacientes. En Cuba los servicios de salud figuran dentro de las premisas principales, por lo que se realiza un esfuerzo constante para el mejoramiento de los hospitales como eje sustancial del sistema de salud cubano.

El Case-Mix, es un término cada vez más utilizado en ambientes de gestión hospitalaria y hace referencia a los distintos tipos de pacientes que se consultan en un hospital, a partir de los mismo se analiza su influencia en el diseño del servicio hospitalario (Hernández Nariño et al. 2014). 
En salud se presenta un número casi ilimitado de variantes tanto en términos de atributos de los grupos poblacionales: edad, sexo, raza, ocupación, cultura, riesgo, como en su diseminación geográfica (universal, regional, sectorial) o en su comportamiento cronológico (estacional, cíclica, explosiva), los que a su vez se combinan entre sí con respecto a esas mismas variables (Hassan Marrero 2018).

Esta herramienta difiere de la manera de gestionar estas instituciones, centrada en aspectos administrativos, por lo que precisa un cambio en la cultura organizacional, al promover la participación y responsabilidad del personal médico en la gestión y la toma de decisiones, más enfocada hacia aspectos clínicos y operativos (Polyzos et al. 2013).

Existen varios sistemas de agrupación de pacientes (Cuadro 1), el objetivo del clasificador, condicionará los criterios de clasificación (consumo de recursos, severidad, pronosticos) utilizados para estructurar el sistema de clasificación. Entre los posibles criterios de clasificación que se proponen para obtener un sistema en función del objetivo para el que se diseñen se encuentran: síntomas, diagnostico, enfermedad, complejidad, pronóstico, severidad, recursos, resultados (García Altés et al. 2000).

La casuística hospitalaria es una de las herramientas más importantes para la Gestión Hospitalaria, según Hernández Nariño (2010) para la puesta en marcha de este sistema de gestión, es preciso disponer de:

Un sistema de agrupación de pacientes que permita definir y clasificar el producto hospitalario. La agrupación de pacientes más universalmente utilizada es la de Categorías Diagnósticas Mayores (CDM), mutuamente excluyentes, según órganos o sistemas afectos, a partir del diagnóstico principal del episodio. En cada CDM suele haber un grupo médico y otro de tipo quirúrgico, denominados como "Otras enfermedades" y "Otros procedimientos quirúrgicos", respectivamente. Estos grupos incluyen diagnósticos o procedimientos que se dan con poca frecuencia y están clínicamente poco definidos.

Cuadro 1. Sistemas de clasificación de pacientes dependientes del diagnóstico médico.

\begin{tabular}{|c|c|}
\hline Sistema de clasificación & Descripción \\
\hline $\begin{array}{l}\text { GRDs (Grupos Relacionados } \\
\text { con el Diagnóstico) o DRG } \\
\text { (Diagnostic Related Groups) } \\
\text { All Patient GRD (AP-GRD) }\end{array}$ & $\begin{array}{l}\text { Sistema de agrupación de pacientes en clases } \\
\text { clínicamente coherentes con igual consumo de } \\
\text { recursos. } \\
\text { Pertenece a la familia de GDR; cubre la atención de } \\
\text { pacientes dentro del hospital como fuera. Incorporan } \\
\text { niveles de gravedad basados en complicaciones y } \\
\text { comorbilidad de los AP GRD. }\end{array}$ \\
\hline $\begin{array}{l}\text { Acute Physiology and Chronic } \\
\text { Health Evaluation (APACHE) }\end{array}$ & $\begin{array}{l}\text { Tiene valor pronóstico y se utiliza sobre todo en } \\
\text { Unidades de vigilancia intensiva. Agrupación según } \\
\text { gravedad (sistema de isogravedad). }\end{array}$ \\
\hline
\end{tabular}


Cuadro 1. Sistemas de clasificación de pacientes dependientes del diagnóstico médico. (continuación)

\begin{tabular}{ll}
\hline Sistema de clasificación & Descripción \\
\hline Disease staging & $\begin{array}{l}\text { Desarrollado para crear grupos homogéneos de } \\
\text { pacientes basados en el diagnóstico principal y } \\
\text { grado de severidad de problemas específicos que } \\
\text { llevan tratamiento médico o quirúrgico. Aplicado a } \\
\text { procesos de hospitalización. }\end{array}$ \\
La unidad de análisis es el paciente y la variable \\
dependiente sería el número de visitas anuales.
\end{tabular}

Fuente: elaboración propia.

Un sistema de información que integre aspectos clínicos y administrativos. La información clínica corresponde a los códigos de la Clasificación Internacional de Enfermedades (C.I.E.10-M.C.), asignados al diagnóstico principal, diagnósticos secundarios, intervenciones quirúrgicas y otros procedimientos médicos; corresponden a la información administrativa, los datos acerca de edad y sexo del paciente, fecha de ingreso y alta, servicio o médico responsable y registro de consumos.

Otros de los sistemas que no se puede dejar de mencionar de gran ayuda, pero solo implementado en los servicios de urgencias, es el Triaje (Hassan Marrero 2018), constituye la puerta de entrada al sistema sanitario (Fernández Landaluce 2020) y el proceso de valoración clínica inicial que clasifica a los pacientes antes de la evaluación diagnóstica y terapéutica médica (Mesquita, Pavlicich, and Luaces 2017), en base a su grado de urgencia, sin necesariamente tomar en cuenta el orden de llegada (Vásquez Alva, Luna Muñoz, and Ramos Garay 2019).

Actualmente se utilizan sistemas de Triaje estructurado en cinco niveles de prioridad, que se asignan asumiendo el concepto de que lo urgente no siempre es grave y lo grave no es siempre urgente y hacen posible clasificar a los pacientes a partir del grado de urgencia, de tal modo que los pacientes más urgentes serán asistidos primero y el resto serán reevaluados hasta ser vistos por el médico (Kripper Mitrano and Saldías Peñafiel 2015).

\section{La gestión hospitalaria en tiempos de covid-19}

La reconversión hospitalaria es una estrategia de gran relevancia en el proceso de contención y tratamiento de una pandemia, que se desarrolló como consecuencia de la experiencia mundial e histórica (Mendoza Popoca and Suárez Morales 2020); no solo se limita a un número de camas mayor o a aumentar la complejidad de estas; se trata además de un conjunto de acciones desplegadas en el Primer Nivel de Atención que 
aumentan su capacidad de identificación y control de casos, educación a la población; mantener servicios para pacientes con otras condiciones agudas y/o crónicas que requieren de un manejo prioritario. Esto en articulación con acciones de reorganización a nivel hospitalario; gestión efectiva de los recursos humanos, suministros y tecnológicos, financieros y; manteniendo las condiciones de protección y seguridad de todo su personal.

Cada institución de salud, deben establecer sus procedimientos de actuación para minimizar los riesgos y mantener los servicios médicos habituales, aunque se encuentren en dimensiones reducidas por la contingencia pandémica, con la garantía de su calidad (Ruiz and Deodato 2020), la pandemia de la COVID-19 ha sido el detonador de múltiples sistemas de actuación en la salud pública a nivel mundial, y en primer lugar el de la gestión hospitalaria para minimizar el impacto en la infraestructura y evitar el colapso hospitalario (Cabrera Solis et al. 2021).

En la mayoría de los países, la respuesta inicial a la pandemia estuvo centrada en acciones curativas, biomédicas y hospitalocéntricas, con un vacío en la atención integral de enfoque promocional/preventivo como primera medida de abordaje (Cabrera Solis et al. 2021), lo que evidencia que el Covid-19 con su vertiginosa expansión, en pocos meses pusiera en evidencia la fragilidad de los mejores sistemas de salud y los mejores modelos de protección social a nivel mundial (Cristaldo et al. 2020).

Los países latinoamericanos contaron con más tiempo para preparar sus sistemas sanitarios, lo cual permitió que anticiparan la compra de los equipos de protección personal, ventiladores e insumos médicos, y comenzaran las obras de acondicionamiento y adecuación de la infraestructura hospitalaria para realizar los test y atender los casos de infectados más severos o en estado crítico (Hernández 2020).

Se fortaleció el sistema de triaje que ha de permitir controlar de forma continua todo lo que pasa dentro y fuera del servicio de emergencia y en las áreas de espera, evaluando periódicamente a los pacientes, garantizando la satisfacción de sus necesidades, controlando los tiempos de espera, dando información fluida a sus familiares y permitiendo la gestión ordenada de la actividad de los diferentes equipos asistenciales. $\mathrm{Su}$ beneficio es mayor en las situaciones en las que la demanda supera a los recursos disponibles (OPPLS); como el caso de la labor del equipo de Medicina Intensiva, o del comité de triaje, que sería identificar de entre los posibles candidatos a aquellos con más opciones de sobrevivir al ingreso en la Unidad de Cuidados Intensivos (de Asúa et al. 2020).

Se ha diseñado un modelo de gestión integral, con tres componentes, que incluye la gestión epidemiológica, la organización de los servicios de salud para la atención preventiva y vigilancia de los casos; esto incluye la implicación en un amplio abanico de iniciativas y prácticas asistenciales derivadas de la nueva demanda asistencial y los nuevos patrones epidemiológicos establecidos por la COVID-19 (Martinez Estalella, Zabalegui, and Guerra 2021). 
En los Hospitales, se crea una entrada separada para todos los casos de pacientes con síntomas de gripe. Al principio, los casos que requerían hospitalización estaban regulados a nivel de institución, pero con el aumento expansivo de la pandemia, fue necesario que las camas fueran reguladas por el gestor Estatal, de forma a atender a todos los municipios del Estado con camas para internación (Ferreira da Silva and Helfenstein Albeirice da Rocha 2021).

En Cuba desde enero de 2020, el Consejo de Ministros aprobó un Plan para la Prevención y Control del Coronavirus. Por eso cuando el 11 de marzo se detectó el primer caso, estaban creadas las condiciones organizativas y materiales imprescindibles. El gobierno convocó a todos en función de ofrecer una respuesta social, científica, política y sanitaria capaz de enfrentar el desafío que la pandemia representa; para ello la totalidad de los centros hospitalarios ha adoptado nuevas medidas en el ámbito de la comunicación interna entre las que destacan la formación interna, el aumento de los equipos de protección, la escucha activa de las propuestas del personal y el apoyo psicológico a los empleados (Castro Martínez and Díaz Morilla 2020).

Los servicios de Cuerpo de Guardia de Pediatría y de Ginecoobstetricia se reubicaron para crear un flujo adecuado de atención a los pacientes infectados por el SARS-Cov-2, sin abandonar la asistencia de urgencias de los pacientes que acudieron a la institución, fundamentalmente en edad pediátrica, con procesos neumónicos no COVID-19, diarreas agudas complicadas con desequilibrios hidroelectrolíticos y minerales y cuadros convulsivos, entre otros; que fueron estabilizados y trasladados a otros centros hospitalarios (Cabrera Solis et al. 2021).

Se ha tenido que recurrir a equipamiento antiguo, junto con respiradores de quirófano, respiradores de transporte, adaptaciones de ventiladores de ventilación no invasiva e incluso anecdóticamente a ambúes mecanizados (Ferrer 2020).

De ahí que la labor preventiva y la pesquisa activa, constituyan herramientas importantes. La aplicación de dicha Tecnología Sanitaria, en esta ocasión, ha sido tal vez la más masiva, continua y sistemática de todas las realizadas hasta el momento; la de mayor participación ciudadana y con el mayor número de pesquisadores (Placeres Hernández 2020).

Los sistemas de clasificación de pacientes cobraron gran relevancia y marcaron un punto de partida en el aumento de la competitividad hospitalaria. Además del triaje, los sistemas de clasificación de pacientes más empleados son GRDs y AP-GRD. La capacidad de infraestructura se vio rápidamente afectada, principalmente los equipos respiradores, se balancea la demanda de los mismos desde otros servicios hacia las salas de atención de pacientes con afecciones respiratorias.

\section{Los indicadores de gestión hospitalaria como signos vitales de la organización}

Toda actividad puede ser medida por medio de indicadores que enfocados a la toma de decisiones sean señales para monitorear la gestión (Castro Rozo 2013). Todo indicador 
debe estar orientado a medir aquellos aspectos claves o factores críticos en los cuales la institución se encuentra interesada en realizar seguimiento y evaluación. Esta situación trae de forma implícita la necesidad de establecer adecuadamente los objetivos estratégicos de la entidad, realizando una identificación clara de aquellas variables que sean relevantes y que se relacionen con los productos estratégicos y los efectos esperados.

Según (Jiménez Paneque 2004) un indicador es una variable que pretende reflejar cierta situación y medir el grado o nivel con que ésta se manifiesta, de manera que resulte útil para evaluar cambios en el tiempo y hacer comparaciones en el espacio.

Para Ruiz Fuentes et al. (2014) los indicadores constituyen un instrumento que permite recoger de manera adecuada y representativa la información relevante respecto a la ejecución y los resultados de uno o varios procesos, de forma que se pueda determinar la capacidad y eficacia de los mismos, así como la eficiencia.

Barbei et al. (2018) define al indicador como la medida que permite el seguimiento y evaluación periódica de las variables clave de una organización mediante su comparación en el tiempo con los correspondientes referentes externos e internos.

Los indicadores deben satisfacer, en la medida de lo posible, las siguientes características:

- Pertinencia: Deben referirse a los procesos y productos esenciales que desarrolla cada institución.

- Independencia: No condicionado a factores externos, tales como la situación del país.

- Confiabilidad: Digno de confianza independiente de quién realice la medición.

- Simplicidad: Debe ser de fácil comprensión, libre de complejidades.

- No redundancia: Debe ser único y no repetitivo.

- Disponibilidad: Los datos básicos para la construcción del indicador deben ser de fácil obtención sin restricciones de ningún tipo.

Los indicadores permiten brindar información para la toma de decisiones, siendo útiles para una amplia gama de usuarios, entre los que se incluyen: gobernantes, funcionarios y ciudadanos (Barbei et al. 2018).

Según López Jara and López Vintimilla (2019b) la utilización de indicadores como herramientas de gestión proporciona grandes ventajas para los gestores y responsables de servicios:

- Facilitar la definición de objetivos al centrarse esta en los indicadores.

- Conocer los resultados y medir la contribución de cada centro al resultado.

- Obtener información sobre las actividades realizadas y, por tanto, diseñar el sistema de información que facilite la toma de decisiones y el control.

- Controlar y mejorar la utilización de los recursos y de las actividades.

- Obtiene una alineación entre las estrategias y el desempeño de los empleados (Romero Cervantes, Vergara Díaz, and Ahumada Arboleda 2015) 
Posibilita la detección de procesos o áreas de la institución en las cuales existen problemas de gestión tales como: uso ineficiente de los recursos, demoras excesivas en la entrega de los productos, asignación del personal a las diferentes tareas (Armijos 2017).

A partir de los conceptos y especificaciones realizadas, el autor resume los indicadores de gestión como una herramienta que permite medir el comportamiento de las variables claves de una organización. Estos brindan información necesaria para la toma de decisiones fundamentales en el cumplimiento de los objetivos, permiten trazar metas alcanzables y controlar de manera óptima el rendimiento de los procesos.

En el servicio de salud, uno de los grandes problemas es la percepción de mala gestión en todos los niveles lo que genera desconfianza e insatisfacción por parte de quienes hacen uso de los servicios de salud. Por ello, los hospitales deben hacer múltiples esfuerzos para garantizar el cumplimiento de aspectos esenciales para su correcto funcionamiento, asegurando la efectividad, eficiencia y eficacia en el desarrollo de sus actividades (Armijos and Núñez Moncada 2020).

En este sentido, es importante llevar a cabo una gestión de recursos eficiente, considerando tanto el recurso humano, como los recursos físicos y económicos. Es por lo que resulta fundamental diseñar, implementar y monitorear indicadores, que permitan conocer la situación actual de la gestión, los procesos y resultados de las organizaciones; y enfocar los esfuerzos sólo a aquellos sectores que requieran mejorar su desempeño mediante el diseño e implementación de un modelo de gestión eficiente (Lagos Ganga 2018).

Estos indicadores deberían ser universales, útiles, sencillos de medir e interpretar, para aprovechar de mejor forma su aplicación. Esto permite además comparar rendimientos con diferentes hospitales públicos dentro de un país, o entre diferentes países de una misma región (Armijos and Núñez Moncada 2020).

En razón a los criterios analizados hasta el momento se hace necesario la elaboración de un modelo de indicadores de gestión que permita a los hospitales, clínicas y centros de salud tanto públicos como privados un control sobre el desempeño en términos de eficiencia y eficacia (López Jara and López Vintimilla 2019a).

(Armijos 2017, Lagos Ganga 2018, Armijos and Núñez Moncada 2020) definen los siguientes grupos de indicadores de gestión hospitalaria (Cuadro 2):

- Indicadores de tiempo: se subdivide en Tiempos de Espera y Tiempos de Proceso. Los Tiempos de Espera comprenden todos aquellos tiempos por los que el paciente ha tenido que esperar para llevar a cabo la atención; mientras que los Tiempos de Procesos, comprenden los tiempos que demora la realización de cada procedimiento necesario para llevar a cabo la atención del paciente.

- Indicadores de calidad: se subdivide en Errores, de cumplimiento de estándares y Satisfacción. Errores, comprende todas aquellas prácticas y/o procedimientos erróneos que se han llevado a cabo en la atención del paciente; mientras que 
Satisfacción, se encarga de medir el cumplimiento de los estándares definidos por la entidad y las exigencias ministeriales correspondientes.

- Indicadores económicos: contempla el gasto real de la institución hospitalaria para la atención a sus pacientes y el cumplimiento con el presupuesto establecido.

- Indicadores de capacidad: comprende la Oferta y Demanda. Oferta, son todos los activos disponibles para llevar a cabo la atención del paciente; mientras que la Demanda, corresponde a la cantidad de pacientes atendidos en la institución.

- Indicadores de eficiencia: se definieron en indicadores de productividad (rendimiento), y cumplimiento de estándares.

Cuadro 2. Indicadores de gestión hospitalaria.

Clasificación Indicadores

Indicadores de Tiempo promedio de espera de admisión, Tiempo promedio de

Tiempo espera triage, Tiempo promedio de espera en box, Tiempo promedio de espera por procedimiento médico, Tiempo promedio de espera por procedimiento de enfermería, Tiempo de espera en consulta externa, Tiempo promedio de espera de traslados, Tiempo promedio de espera de traslados, Tiempo promedio de espera de actividades de apoyo al diagnóstico, Tiempo promedio de espera de alta médica, Tiempo promedio de espera de resultados de actividades de apoyo, Tiempo promedio admisión, Tiempo promedio de triage, Tiempo promedio de procedimiento médico, Tiempo promedio de procedimiento de enfermería, Tiempo promedio de actividades de apoyo, Tiempo promedio del ciclo del paciente, Tiempo promedio de atención médica.

Indicadores de Porcentaje de infecciones intrahospitalarias, Tasa de mortalidad en calidad el servicio de urgencias, Tasa neta de mortalidad hospitalaria, Tasa de pacientes fallecidos en espera por hospitalización, Tasa de reclamos realizados por los pacientes, Porcentaje de reingresos hospitalarios, Tasa de eventos adversos relacionado con la mala identificación de pacientes, Permanencia superior a 24 horas en los servicios de urgencias, Porcentaje de errores en la medicación, Tiempo de demora en intervenciones quirúrgicas urgentes, Número de pacientes en lista de espera quirúrgica, Retorno a urgencias en 72 horas, Existencia de protocolos clínicos, Tasa de satisfacción del paciente, Tasa de satisfacción de los profesionales, Porcentaje de cirugías suspendidas, Encuesta de satisfacción de los pacientes, Encuesta de satisfacción de los profesionales.

Indicadores Porcentaje de pérdidas de citas, Costo promedio por paciente, económicos Ejecución presupuestaria, Costo promedio por servicio.

Indidadores de Promedio diario de camas disponibles, Intervalo de sustitución cama, Capacidad Promedio de días de estancia, Índice de rotación o giro de camas, Intervalo de sustitución cama, Promedio de censo diario, Tasa de pacientes mañana, Tasa de pacientes tarde, Tasa de pacientes noche, Capacidad de quirófanos.

Indicadores de Rendimiento hora-médico, Productividad hora-médico, Promedio eficiencia de estancia, Rendimiento de la sala de operaciones, Rendimiento de la sala de operaciones, Porcentaje de cirugías ambulatorias, 


\begin{tabular}{ll}
\multicolumn{2}{c}{ Cuadro 2. Indicadores de gestión hospitalaria. (continuación) } \\
\hline Clasificación & Indicadores \\
\hline $\begin{array}{l}\text { Indicadores de } \\
\text { eficiencia }\end{array}$ & $\begin{array}{l}\text { Rendimiento cama, Relación enfermeras / médicos, Número de } \\
\text { camas por médico, Tasa de ausentismos. }\end{array}$ \\
\hline
\end{tabular}

Fuente: Elaboración propia

Estos indicadores están orientados a la mejora, son fáciles de interpretar, y pueden ser medidos sin grandes recursos, sin embargo, requieren de sistemas de información que apoyen la recolección de datos (Armijos and Núñez Moncada 2020).

Es de gran importancia el manejo de estos indicadores, ya que permiten medir el desempeño de la institución para poder controlarla. Esta información documentada deber ser analizada, procesada y dada a conocer de una forma visual y práctica, representada en un cuadro de mando de indicadores de gestión, para que pueda ser interpretada fácilmente por los integrantes de la compañía, empleando dicha información como una guía para la toma de decisiones y acciones correctivas en aquellas áreas que no estén cumpliendo con los objetivos planteados (Vélez Ochoa 2019).

La recopilación de información a través de estos indicadores da luces respecto de la situación de la institución. En este punto es fundamental recalcar la importancia del compromiso de todos los niveles que conforman la organización, de ellos dependerá: la veracidad de los datos, la confiabilidad y oportunidad de la información y el uso adecuado de estos indicadores para tomar acciones específicas de mejora del desempeño organizacional (Armijos and Núñez Moncada 2020).

La gestión hospitalaria es vital importancia si se quieren ofrecer servicios de alta calidad. La actual pandemia mundial provocada por el covid 19 obliga a las instituciones hospitalarias a ajustar los recursos físicos y humanos con el objetivo de satisfacer las necesidades de los usuarios, es por ello, por lo que debe existir una visión integrada, tanto de los procesos asistenciales, como de los procesos administrativos para el adecuado funcionamiento de las organizaciones, de ahí, que los indicadores de gestión hospitalaria jueguen un papel fundamental como herramienta de gestión.

\section{Conclusiones}

- Con la aparición de la covid 19 en diciembre del 2019 el sistema sanitario mundial se vio ante una cruzada inigualable. Los servicios de salud se vieron colapsados rápidamente debido al fácil contagio y las características clínicas de la enfermedad. La gestión hospitalaria como servicio responsable de cubrir con el $90 \%$ de la demanda que la atención primaria no puede cubrir.

- El nuevo sistema de gestión integrada contiene tres pilares principales la gestión epidemiológica, de servicios preventivos y vigilancia de caso, para lo que se ha empleado alternativas como la pesquisa activa y las encuestas epidemiológicas apoyadas en aplicaciones informáticas.

- Los indicadores brindan información que permite enfocar al hospital en medidas correctivas o mejoras en procesos para lograr el cumplimiento de sus objetivos. 
Esta propuesta de indicadores es una guía para mejorar la toma de decisiones en hospitales públicos fomentando mejoras en el desempeño y el uso eficiente de los recursos. Los indicadores se pueden clasificar en: indicadores de tiempo, indicadores de calidad, indicadores económicos, indidadores de capacidad, indicadores de eficiencia.

\section{Referencias bibliográficas}

Amador Guevara, José. 1979. "Hospital y Salud Pública." Revista Médica de Costa Rica XLVI (469):179-181. https://www.google.com/url?sa=t\&source=web\&rct=j\&url=https://www.binasss .sa.cr/bibliotecas/bhp/cupula/v5n8/art3.pdf\&ved=2ahUKEwieuaC9qdvvAhUEI KwKHfq2CAgQFjALegQINRAF\&usg=AOvVaw12hP7yxek4Iw5LyxtX872H.

Armijos, Juan Carlos. 2017. "Diseño e implementación de un modelo de indicadores de gestión para evaluar el desempeño de hospitales públicos."Tesis para optar al grado de MAGÍSTER EN CONTROL DE GESTIÓN, Universidad de Chile.

Armijos, Juan Carlos, and Alicia Núñez Moncada. 2020. "Indicadores de gestión para evaluar el desempeño de hospitales públicos: Un caso de estudio en Chile y Ecuador." Rev Med Chile 148 (5):ISSN 626-643. doi: http://doi.org/10.4067/S0034-98872020000500626.

Barbei, Alejandro Agustín, Graciela Neira, Paula Carolina González, and Florencia Zinno Arbio. 2018. "Indicadores de gestión en las entidades públicas." XII Congreso Iberoamericano de Contabilidad de Gestión y IV Congreso Internacional de Gestión Empresarial. Quito, Ecuador. http://sedici.unlp.edu.ar/handle/10915/68036.

Cabrera Solis, Lucrecia , Sara Amneris Urgellés Carreras, Clara Ligia Santamaría Trujillo, Guillermo Rodríguez Iglesias, and Eneida Gil Agramonte. 2021. "Gerencia del Centro Materno-Infantil para el enfrentamiento a la COVID-19. Hospital Dr. Luis Díaz Soto." Revista de Información científica para la Dirección en Salud. INFODIR (35). ISSN 1996-3521. http://revinfodir.sld.cu/index.php/infodir/article/view/1038.

Castro Delgado, Rafael , Pedro Arcos González, and Alberto Rodríguez Soler. 2009. "Sistema sanitario y triaje ante una pandemia de gripe: un enfoque desde la salud pública." Emergencias 21:376-381. https://scholar.google.es/scholar?q=Sistema+sanitario+y+triaje+ante+una+pand emia+de+gripe:+un+enfoque+desde+la+salud+p\%C3\%BAblica.\&hl=es\&as_sdt $=0,5$.

Castro Martínez, Andrea, and Pablo Díaz Morilla. 2020. "Gestión de la comunicación interna y externa en instituciones hospitalarias privadas en España durante la crisis 
del COVID-19." Revista de Comunicación y Salud 10 (2):525-547. 2174-5323. doi: https://doi.org/10.35669/rcys.2020.10(2).525-547.

Castro Rozo, Fabio Enrique 2013. "Indicadores de gestión: toma de decisiones basada en inteligencia de negocios." Tecnología Investigación y Academia 1 (2). 23448288. https://revistas.udistrital.edu.co/index.php/tia/article/view/4639.

Chavarro Carvajal, Diego Andrés, Luis Carlos Venegas Sanabria, Ronald Camilo Gómez Arteaga, Sandra Milena Caicedo Correa, and Carlos Alberto Cano Gutiérrez. 2020. "Retos de la atención a las personas mayores con COVID-19 a nivel hospitalario." Revista ACGG $34 \quad$ (1):81-86. $\quad$ ISSN $\quad 0122-6916$. https://repository.javeriana.edu.co/handle/10554/53617.

Cristaldo, Patricia, Rosa Ruffinelli, Belinda García, Álvaro Javier Lo Bianco, and Felicina Meza. 2020. "La pandemia no tiene fronteras. Acercamiento a la realidad de ciudades gemelas Foz de Iguazu/Ciudad del Este." Revista MERCOSUR de Políticas Sociales 4:78-90. ISSN 2663-2047. doi: https://doi.org/10.28917/ism.2020-v4-78.

de Asúa, Diego Real, Jose María Galván, Judit Iglesias, Julia Fernández, Diego Rodríguez, Patricia Albert, Rosa Méndez, Tamara Alonso, María Churruca, and Jorge Salamanca. 2020. "Criterios de triaje para reanimación cardiopulmonar y soporte vital avanzado durante la epidemia COVID-19." Medicina Clínica 155 (5):215-219. ISSN 0025-7753. doi: http://doi.org/10.1016/j.medcli.2020.04.009.

Fernández Landaluce, Ana 2020. "Triaje de Urgencias de Pediatría." Protocolos diagnósticos y terapéuticos en Urgencias de Pediatría 1:1-13. https://scholar.google.es/scholar?hl=es\&as_sdt=0\%2C5\&q=Triaje+de+Urgencia $\mathrm{s}+\mathrm{de}+$ Pediatr\%C3\%ADa.\&btnG=.

Ferreira da Silva, Ruy, and Marceli D Helfenstein Albeirice da Rocha. 2021. "La pandemia en un hospital universitario en el norte del Brasil." Revista Subjetividad y Procesos Cognitivos $24 \quad$ (2). 1852-7310. https://publicacionescientificas.uces.edu.ar/index.php/subyprocog/article/view/1 064.

Ferreira Junior, Roberto Rodney, and Adelino Paulo Porto. 2018. "La calidad de vida en el trabajo y el ausentismo como indicadores de resultado de gestión hospitalaria." Ciencias Administrativas 6 (11). 2314-3738. http://revistas.unlp.edu.ar/CADM.

Ferrer, Ricard. 2020. "Pandemia por Covid-19: el mayor reto de la historia del intensivismo." Medicina intensiva 44 (6):323-324. doi: http://doi.org/10.1016/j.medin.2020.04.002.

Fuentelsaz Gallego, Carmen, Teresa Moreno Casbas, David López Zorraquino, Teresa Gómez García, and Esther González María. 2012. "Percepción del entorno laboral de las enfermeras españolas en los hospitales del Sistema Nacional de Salud. 
Proyecto RN4CAST-España." Enfermería Clínica 22 (5):261-268. 1130-8621. doi: http://doi.org/10.1016/j.enfcli.2012.09.001.

García Altés, Anna, F Martínez, E Carrillo, and S Peiró. 2000. "Sistemas de clasificación de pacientes en centros de media y larga estancia: evolución y perspectivas de futuro." Gaceta Sanitaria $14 \quad$ (1):48-57.

0213-9111. http://zl.elsevier.es/es/revista/gaceta-sanitaria138/articulo/sistemas-clasificacionpacientes-centros-media-13008589.

González Pérez, R. 1997. "Modelo GOS para la mejora de la gestión de operaciones de servicio. Aplicación parcial en servicios hospitalarios."Tesis presentada en opción al titulo científico de Master en Ciencias, Universidad de Matanzas.

Hassan Marrero, Nadima. 2018. "Mejora de la gestión del flujo de pacientes en el Hospital Clínico Quirúrgico Docente Faustino Pérez Hernández."Tesis presentada en opción al grado científico de Máster. Mención Gestión de la Producción y los Servicios Departamento de Ingeniería Industrial Universidad de Matanzas

Hernández Aguado, Ildefonso, and Ana M García. 2021. "¿Será mejor la salud pública tras la COVID-19?" Gaceta Sanitaria 35 (1):1-2. ISSN 0213-9111. doi: http://doi.org/10.1016/j.gaceta.2020.06.004.

Hernández Nariño, Arialys. 2010. "Contribución a la gestión y mejora de procesos en instalaciones hospitalarias del territorio matancero. ."Tesis presentada en opción al Grado Científico de Doctor en Ciencias Técnicas Departamento de Ingeniería Industrial Universidad de Matanzas "Camilo Cienfuegos"

Hernández Nariño, Arialys, Alberto Medina León, Dianelys Nogueira Rivera, Ernesto Negrín Sosa, and Maylin Marqués León. 2014. "La caracterización y clasificación de sistemas, un paso necesario en la gestión y mejora de procesos. Particularidades en organizaciones hospitalarias." Dyna 81 (184):193-200. 0012-7353. http://www.redalyc.org/articulo.oa?id=49630405027.

Hernández, René A. 2020. "Covid-19 y América Latina y el Caribe: los efectos económicos diferenciales en la región." Instituto Universitario de Análisis Económico y Social:50. ISSN 2172-7856. http://hdl.handle.net/10017/43143.

Jiménez Paneque, Rosa E. 2004. "Indicadores de calidad y eficiencia de los servicios hospitalarios: Una mirada actual." Revista cubana de salud pública 30 (1):0-0. ISSN 0864-3466. http://scielo.sld.cu/scielo.php?pid=S0864$34662004000100004 \&$ script=sci_arttext\&tlng=pt.

Kripper Mitrano, Cristóbal, and Fernando Saldías Peñafiel. 2015. "Estratificación de riesgo (Triage) en el Servicio de Urgencia." Revista Chilena de Medicina Intensiva 
https://scholar.google.es/scholar?hl=es\&as_sdt=0\%2C5\&q=Estratificaci\%C3\% $\mathrm{B} 3 \mathrm{n}+\mathrm{de}+$ riesgo+\%28Triage\%29+en+el+Servicio+de+Urgencia.\&btnG $=$.

Lagos Ganga, Javiera. 2018. "Medición del desempeño y propuestas de mejora en unidades de emergencia de salud privada."Tesis para optar al grado de Magíster en Control de Gestión GESTIÓN, Universidad de Chile.

León Moyal, H. . 2021. "Funciona nuevo laboratorio de Biología Molecular en Guantánamo." Juventud Rebelde. http://www.juventudrebelde.cu/cuba/2021-0222/funciona-nuevo-laboratorio-de-biologia-molecular-en-guantanamo.

López Jara, Ana Alexandra, and Xavier Raúl López Vintimilla. 2019b. "Indicadores para evaluar la eficiencia hospitalaria. Caso de estudio: Clínica privada de la ciudad de Macas Indicators to evaluate hospital efficiency. Case study: Private clinic in the city of Macas." EPISTEME 6 (3):383-398 ISSN 1390-9150. https://scholar.google.es/scholar?hl=es\&as_sdt=0\%2C5\&q=Indicadores+para+e valuar+la+eficiencia+hospitalaria.+Caso+de+estudio\%3A+Cl\%C3\%ADnica+pri vada + de + la + ciudad + de + Macas $\& b t n G=$.

Marqués León, Maylín. 2013. "Modelo y procedimientos para la planificación de medicamentos y materiales de uso médico en instituciones hospitalarias del territorio matancero. ."Tesis presentada en opción al grado científico de Doctor en Ciencias Técnicas Departamento de Ingeniería Industrial Universidad de Matanzas "Camilo Cienfuegos".

Martinez Estalella, Gemma, Adelaida Zabalegui, and Sonia Sevilla Guerra. 2021. "Gestión y liderazgo de los servicios de Enfermería en el plan de emergencia de la pandemia COVID-19: la experiencia del Hospital Clínic de Barcelona." Enfermería Clínica 31 (1):S12-S17. ISSN 1130-8621. doi: https://doi.org/10.1016/j.enfcli.2020.05.002.

Mendoza Popoca, Cecilia Úrsula, and Mario Suárez Morales. 2020. "Reconversión hospitalaria ante la pandemia de COVID-19." Revista Mexicana de Anestesiología https://scholar.google.es/scholar?hl=es\&as_sdt=0\%2C5\&q=Reconversi\%C3\%B $3 \mathrm{n}+$ hospitalaria+ante+la+pandemia+de+COVID-19.\&btnG $=$.

Mesquita, Mirta, Viviana Pavlicich, and Carlos Luaces. 2017. "El sistema español de triaje en la evaluación de los neonatos en las urgencias pediátricas." Revista Chilena de Pediatría 88 (1):107-112. doi: http://doi.org/10.1016/j.rchipe.2016.07.00.

Morales Carrillo, Ángela Esperanza, Manuel Felipe Vega Novoa, Manuel Ángel Camacho Oliveros, and Milton Januario Rueda Varón. 2018. "Caracterización de los procesos de atención y flujo de pacientes de la ruta integral de atención en salud materno-perinatal: caso red pública hospitalaria del norte de 
Cundinamarca." Avances: Investigacion en Ingeniería 15 (1):30-47. 1794-4953. doi: http://doi.org/10.18041/1794-4953/avances.1.1651.

MSP. 2019. "Anuario estadístico de salud 2019." Dirección de Registros Médicos y Estadísticas de Salud. 1561-4433. http://bvscuba.sld.cu/anuario-estadisticodecuba/.

OIT. 2019. "Panorama Laboral 2019: América Latina y el Caribe. Panorama laboral America Latina y el Caribe." https://www.ilo.org/wcmsp5/groups/public/---americas/---rolima/documents/publication/wcms_732198.pdf.

OPS. 2017. "Programa de Líderes en Salud Internacional Dr. Edmundo Granda Ugalde. PLSI 2010." Organización Panamericana de la Salud. http://www.paho.org/lideresensaludinternacional.

Peñafiel Chang, Luis, Gisella Camelli, and Paolo Peñafiel Chang. 2020. "Pandemia COVID-19: Situación política-económica y consecuencias sanitarias en América Latina." CIENCIA UNEMI 13 (33):120-128. 2528-7737. doi: https://doi.org/10.29076/issn.2528-7737vol13iss33.2020pp120-128p.

Placeres Hernández, José Fernando. 2020. "Pesquisa activa, contribución desde la Atención Primaria de Salud para el control de la COVID-19." Revista Médica $\begin{array}{lllll}\text { Electrónica } & 42 & (4): 2148-2159 . & \text { ISSN }\end{array}$ http://scielo.sld.cu/scielo.php?script=sci_arttext\&pid=S168418242020000402148.

Polyzos, Nikolaos, Haralampos Karanikas, Eleftherios Thireos, Catherine Kastanioti, and Nick Kontodimopoulos. 2013. "Reforming reimbursement of public hospitals in Greece during the economic crisis: implementation of a DRG system." Health policy 109 (1):14-22. doi: https://doi.org/10.1016/j.healthpol.2012.09.011.

Romero Cervantes, Sergio Andrés, Andrés Vergara Díaz, and Pedro Peter Ahumada Arboleda. 2015. "Indicadores de gestión un reto en la competitividad de las organizaciones." Liderazgo Estratégico 5 (1):124-134. ISSN 2463-0217. http://revistas.unisimon.edu.co/index.php/liderazgo/article/view/3243.

Ruiz, Ana Paula, and Sérgio Deodato. 2020. "Percepción de las enfermeras gestoras sobre su gestión y liderazgo en tiempos de pandemia: una revisión de alcance." Enfermería Activa 6:33. ISSN 2444-782X. https://scholar.google.es/scholar?hl=es\&as_sdt=0\%2C5\&q=Percepci\%C3\%B3n + de+las+enfermeras+gestoras+sobre+su+gesti\%C3\%B3n+y+liderazgo+en+tiem pos+de+pandemia\%3A+una+revisi $\% \mathrm{C} 3 \% \mathrm{~B} 3 \mathrm{n}+\mathrm{de}+\mathrm{alcance} \& \mathrm{btnG}=$.

Ruiz Fuentes, Daysi, Rosa Mercedes Almaguer Torres, Isabel Cristina Torres Torres, and Alejandro Miguel Hernández Peña. 2014. "La gestión por procesos, su surgimiento y aspectos teóricos." Ciencias Holguín 20 (1):1-11. 1027-2127. 
https://scholar.google.es/scholar?hl=es\&as_sdt=0\%2C5\&q=La+gesti\%C3\%B3n + por+procesos $\% 2 \mathrm{C}+$ su+surgimiento $+\mathrm{y}+$ aspectos+te $\% \mathrm{C} 3 \% \mathrm{~B} 3$ ricos. $\& \mathrm{btnG}=$.

Sánchez Torres, Diana Alejandra. 2017. "Accesibilidad a los servicios de salud: debate teórico sobre determinantes e implicaciones en la política pública de salud." Revista Médica del Instituto Mexicano del Seguro Social 55 (1):82-89. 04435117. http://www.redalyc.org/articulo.oa?id=181529929002.

Suárez Barraza, Manuel F. 2020. "Implementación del "Kaizen-Innovación de ProcesosJidoka" para hacer frente al COVID-19: un caso de estudio en un hospital público." Ingeniería Industrial (039):75-96. 2523-6326. doi: http://doi.org/10.26439/ing.ind2020.n039.4916.

Terán Rosero, Gustavo Javier, Edwin Jonathan Mora Chuquer, Milena del Rocío Gutiérrez Villarrea, Sonia Cumanda Maldonado Tapia, Wilmar Alberto Delgado Campaña, and Angie Fernández Lorenzo. 2019. "La gestión de la innovación en los servicios de salud pública." Revista Cubana de Investigaciones Biomédicas 36

ISSN

1561-3011. http://www.revibiomedica.sld.cu/index.php/ibi/article/view/81.

Vásquez Alva, Rolando, Consuelo Luna Muñoz, and Cleto M Ramos Garay. 2019. "El triage hospitalario en los servicios de emergencia." Revista de la Facultad de Medicina Humana 19 (1):5-5. 2308-0531. doi: http://doi.org/.25176/RFMH.v19.n1.1797.

Vélez Ochoa, Andrés Ricardo. 2019. "Diseño de un cuadro de mando de indicadores de gestión para el Hospital León Becerra de la ciudad de Guayaquil."Tesis Doctoral, Universidad de Guayaquil. Facultad de Ingeniería Industrial.

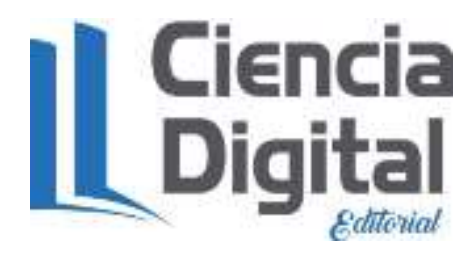




\section{PARA CITAR EL ARTÍCULO INDEXADO.}

Sánchez Suárez, Y., Trujillo García, L., Marqués León, M., \& Santos Pérez, O. (2021). Los indicadores de gestión hospitalaria en tiempos de Covid 19. Visionario Digital, 5(4), 58-77. https://doi.org/10.33262/visionariodigital.v5i4.1901

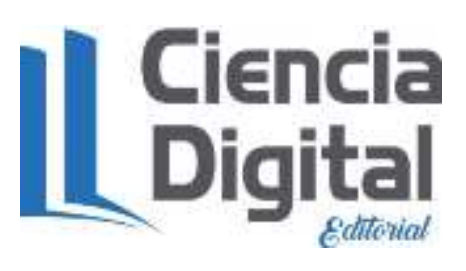

El artículo que se publica es de exclusiva responsabilidad de los autores y no necesariamente reflejan el pensamiento de la Revista Visionario Digital.

El artículo queda en propiedad de la revista y, por tanto, su publicación parcial y/o total en otro medio tiene que ser autorizado por el director de la Revista Visionario Digital.
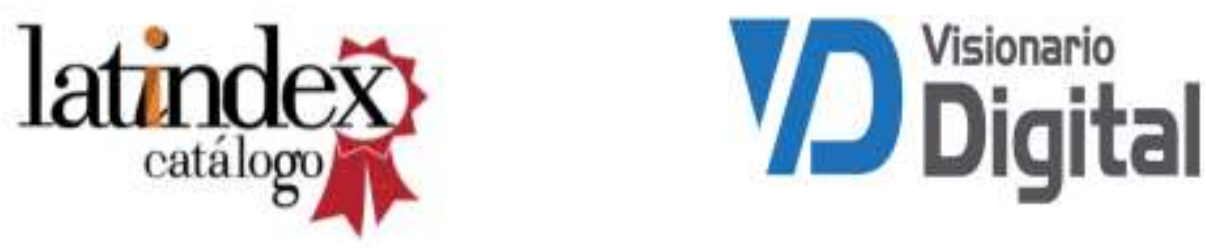